\title{
Porpora trombotica trombocitopenica (PTT) o sindrome di Moschowitz: una vera urgenza ematologica
}

\author{
Thrombotic thrombocytopenic purpura (TTP) or Moschowitz syndrome: \\ a true hematologic emergency
}

\section{Deborah Melis $^{a}$, Gianluca Michelis ${ }^{b}$, Marcello Brignone ${ }^{b}$, Marina Cavaliere ${ }^{b}$, Rodolfo Tassara ${ }^{b}{ }^{*}$}

a Scuola di Specializzazione in Medicina Interna, Università degli Studi di Torino

b SC Medicina Interna (Direttore: Dr. Rodolfo Tassara), ASL 2 Savonese, Ospedale "San Paolo", Savona

Ricevuto l'8 gennaio 2010; accettato il 7 maggio 2010

disponibile online il 5 agosto 2010

\section{KEYWORDS \\ Thrombotic thrombocytopenic purpura; Hemolytic anemia; Thrombocytopenia; Thrombotic microangiopathy.}

\begin{abstract}
Summary
Introduction: Thrombotic thrombocytopenic purpura (TTP) is a thrombotic microangiopathy caused by congenital or inherited disorders involving the processing of the ultra-large forms of von Willebrand factor. As a result, platelet-rich microthrombi form in the small arterial vessels of various organs, particularly those of the brain, heart, and kidneys. The idiopathic autoimmune form of TTP is the most common. There are various subgroups of acquired TTP associated with HIV infection, sepsis, pregnancy, autoimmune disease, various disseminated malignancies, and drugs. If not promptly treated, TTP is associated with high mortality, making it a true medical emergency.

Materials and methods: The article is based on a review of the literature published between January and October of 2009. Its aim is to clarify the diagnosis, treatment, and follow-up of TTP. Results: Diagnostic criteria include the presence of microangiopathic hemolytic anemia associated with thrombocytopenia in the absence of other obvious causes. Assays of ADAMTS13 activity and titration of acquired antibodies against this enzyme are indicated in the follow-up of disease and as prognostic indicators. Treatment centers around daily plasma exchange associated with immunosuppressant drug therapy, particularly steroids and more recently the monoclonal anti-CD20 antibody rituximab.

Discussion: Despite improved treatment, TTP is still associated with significant mortality $(10-20 \%)$, particularly when plasma exchange is initiated late. Relapse also occurs in a
\end{abstract}

\footnotetext{
* Corrispondenza: Dipartimento di Medicina Interna, via Genova 30 - 17100 Savona.

E-mail: r.tassara@asl2.liguria.it (R. Tassara).
} 
substantial proportion of patients $(10-40 \%)$ although the frequency of this outcome may be reduced by rituximab therapy.

(c) 2010 Elsevier Srl. All rights reserved.

\section{Introduzione}

\section{Caso clinico}

Paziente femmina, 38 anni, anamnesi patologica remota negativa per patologie di rilievo. In terapia con estroprogestinico. Due gravidanze a termine. Giunge alla nostra osservazione per la comparsa di ecchimosi alle braccia associate a piastrinopenia e febbre. I primi esami di laboratorio escludono una forma autoimmune di piastrinopenia. Successive indagini confermano la presenza di anemia emolitica, insufficienza renale moderata e progressivo peggioramento della trombocitopenia. Allo striscio periferico, evidenza di rarissimi schistociti. Nel sospetto di porpora trombotica trombocitopenica (PTT), si decide di iniziare la plasmaferesi, con rapido benefico.

La paziente presenta, in quarta giornata dal ricovero, una severa crisi ipertensiva che richiede il trasferimento in Terapia Intensiva. Un ecocardiogramma mostra una grave cardiomiopatia ipertrofica. Lo studio per la ricerca di eventuali forme secondarie di ipertensione fornisce esito negativo, mentre la TAC e la RMN dell'encefalo evidenziano la presenza di pregresse lesioni ischemiche cerebrali. Lo screening immunologico risulta negativo. I test microbiologici su sangue e urine danno esito negativo; si considera, tuttavia, la precoce somministrazione di terapia antibiotica ad ampio spettro per la presenza di una leucocitosi neutrofila e anomalie all'esame chimico-fisico delle urine. Il dosaggio dell'attività di ADAMTS13 e la determinazione del titolo anticorpale, eseguiti durante la terapia aferetica e a distanza di 3 mesi dalla dimissione, risultano nella norma.

La paziente è stata dimessa in buone condizioni generali, in terapia con antipertensivi e antiaggregante piastrinico.

La paziente ha fornito il consenso informato.

La PTTè una sindrome acuta caratterizzata dalla presenza di anemia emolitica microangiopatica e piastrinopenia.

Diversi studi hanno cercato di stimare l'incidenza annua di PTT [1-3]. Nel Registro dell'Oklahoma, in cui la diagnosi di PTT è stata confermata dal dosaggio di ADAMTS13, l'incidenza è di 1,74 casi per milione anno-persona. Nonostante entrambi i sessi ne siano colpiti, questa patologia è più frequente nelle donne ( 2 casi su 3 ); il picco di incidenza si verifica in un'età compresa fra i 30 e i 50 anni. Il rischio per $i$ soggetti di razza nera è 9 volte più alto.

In assenza di trattamento, la mortalità supera il $90 \%$, mentre si riduce al $10-20 \%$ dopo adeguata terapia con plasmaferesi o infusione di plasma. Tuttavia, la metà dei decessi è attribuibile alle complicanze associate alla plasmaferesi e all'ospedalizzazione (sepsi, emorragie, trombosi) [4].

\section{Cenni storici}

Il primo caso di PTT fu descritto nel 1924 da Moschowitz [5]. Tuttavia, la patogenesi della PTT rimase misconosciuta sino alla fine degli anni Ottanta del secolo scorso.
Nel 1982 Moake et al. [6] osservarono, nel plasma dei pazienti affetti da PTT cronica ricorrente, multimeri a elevato peso molecolare del fattore di von Willebrand (VWF), una glicoproteina presente a livello di cellule endoteliali, piastrine e plasma, definiti "ultralarge" (ULVWF). Gli ULVWF promuovono l'aggregazione piastrinica e la trombosi microvascolare piastrino-dipendente, suggerendo la carenza, nei soggetti con PTT, della proteasi del VWF in grado di degradare queste forme multimeriche.

Nel 1996, Furlan et al. [7] e Tsai [8] isolarono una proteasi del VWF il cui deficit era associato alla PTT e dimostrarono che livelli plasmatici bassi o non dosabili di questa proteasi erano indicatori specifici di PTT $[9,10]$. La proteasi del VWF, identifica e purificata nel 2001 [11-13] e oggi nota come ADAMTS13, appartiene alla famiglia delle metalloproteasi ADAMTS (A Disintegrin And Metalloprotease with ThromboSpondin-1 repeats).

Nel 2001 Levy et al. [14] identificarono il gene codificante ADAMTS13 e descrissero, in 7 famiglie indipendenti, 12 differenti mutazioni, evidenziando il difetto molecolare responsabile della rara forma di PTT congenita. In un'alta percentuale di pazienti affetti da PTT idiopatica, che presentavano un deficit di ADAMTS13, sono stati identificati anticorpi di classe IgG inibenti la metalloproteasi direttamente o causandone la rimozione dal circolo $[9,10]$.

\section{Patogenesi}

In condizioni di elevato shear nel flusso sanguigno, i multimeri ULVWF secreti dalle cellule endoteliali attivate sono ancorati come filamenti alle molecole di P-selectina esposte sulla superficie dell'endotelio attivato. ADAMTS13 si lega ai domini A3 accessibili del VWF e degrada i multimeri ULVWF mediante il taglio del legame peptidico tra i residui Tyr1605Met1606 localizzati nel dominio A2. Come conseguenza della carenza di ADAMTS13, i multimeri ULVWF non vengono degradati dopo esser stati secreti, ma rimangono ancorati all'endotelio. Le piastrine circolanti aderiscono, attraverso la glicoproteina Gplb e il complesso Gpllb/IIla, ai domini A1 e A3 delle subunità monomeriche dei filamenti ULVWF ancorati alla P-selectina e formano grossi trombi, potenzialmente occlusivi, a livello delle arteriole terminali e dei capillari di diversi organi (cuore, pancreas, reni, surreni, cervello, milza e fegato), risparmiando il circolo polmonare (fig. 1).

Ancora in studio sono altre ipotesi patogenetiche, in particolare nei pazienti che non presentano un deficit di ADAMTS13: danno endoteliale, aumentata aggregazione piastrinica, fattori genetici.

La PTT, come altri disordini vascolari e trombotici, sembra essere una malattia multifattoriale: gli stress acuti, inducendo la liberazione di citochine infiammatorie o altri mediatori protrombotici, potrebbero scatenare o indurre un singolo evento o una recidiva probabilmente alterando l'equilibrio tra i livelli del VWF e l'attività di ADAMTS13 nei soggetti più suscettibili. 


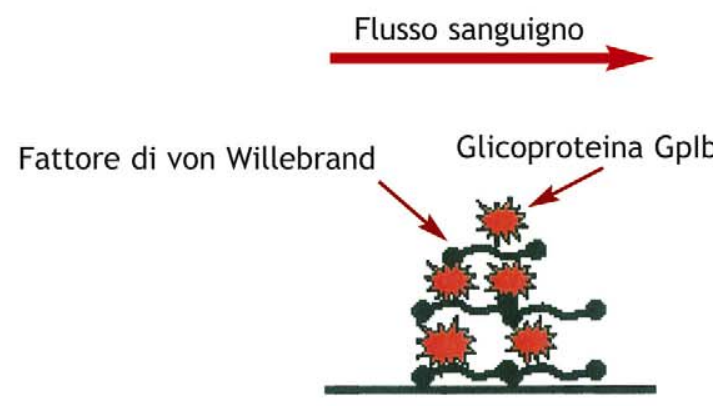

Parete vasale

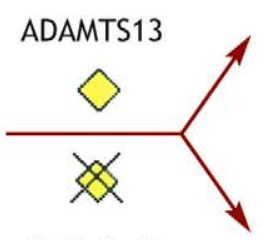

Deficit di ADAMTS13

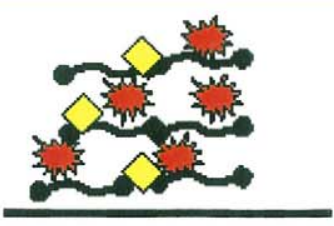

Emostasi normale

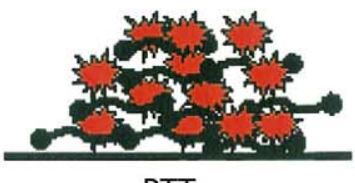

PTT

Figura 1 Patogenesi della PTT in seguito a deficit di ADAMTS13. Fonte: modificata da Sadler JE. Blood 2008;112(1):11-8.

\section{Classificazione}

Esistono due differenti forme di PTT: congenita e acquisita (tabella 1).

La PTT congenita è causata da mutazioni a carico del gene localizzato sul cromosoma 9q34 che codifica per ADAMTS13; può presentarsi in omozigosi, eterozigosi composta o con un singolo polimorfismo e si manifesta prevalentemente, ma non esclusivamente, alla nascita o nel corso dell'infanzia [14]. A oggi sono state identificate 60 mutazioni: il $60 \%$ è costituito da mutazioni missens, il rimanente $40 \%$ da mutazioni nonsens, frameshift o mutazioni di splicing.

La PTT acquisita può essere distinta in due tipi:

- idiopatica, immunomediata, conseguente alla presenza di autoanticorpi che inibiscono l'attività proteolitica di ADAMTS13 e/o si legano alla proteasi accelerandone la clearance plasmatica [15];

- secondaria, riconducibile a una massiva stimolazione dell'endotelio con un rilascio di multimeri ULVWF eccedente la capacità di degradazione da parte della proteasi.

Entrambe le forme sono normalmente scatenate da situazioni fisiologiche o patologiche che causano una massiva attivazione endoteliale.

Diverse sono le cause predisponenti e/o concomitanti di PTT secondaria (tabella 2). È frequente l'associazione con adenocarcinoma mucinoso, metastasi o una neoplasia occulta. In tali casi la presentazione clinica è atipica e vi è una mancata risposta alla plasmaferesi.

Tra i farmaci si segnala la chinidina, presente in molti integratori alimentari e bevande. Può essere sufficiente una sola esposizione, con meccanismo idiosincrasico, per indurre la produzione di anticorpi verso piastrine, neutrofili, linfociti, globuli rossi e cellule endoteliali. La chinidina sembra essere il farmaco più spesso associato allo sviluppo di PTT. Si riscontra, tuttavia, una buona risposta alla sospensione dell'assunzione e alla plasmaferesi [16].

Per i chemioterapici, la tossicità è dose-dipendente, a esordio insidioso, anche a distanza di mesi dal termine della terapia. Non sono disponibili evidenze sulla risposta alla plasmaferesi.

Anche gli antiaggreganti sembrano coinvolti nello sviluppo di PTT secondaria. La ticlopidina, in particolare, indurrebbe un aumento del rischio di 200-500 volte [17]. Il trattamento con plasmaferesi sembrerebbe ridurre la mortalità, ma mancano studi controllati. Al contrario, il clopidogrel non aumenterebbe il rischio di sviluppare PTT [18].

La PTT può costituire la presentazione iniziale in pazienti affetti da HIV. Il meccanismo appare legato a un danno diretto dell'endotelio, da parte del virus, che induce una riduzione nella sintesi di ADAMTS13. Sono state osservate una remissione della PTT nei pazienti trattati con la "Highly Active Antiretroviral Therapy" (HAART) e una recidiva nel

Tabella 1 Classificazione e sottotipi di PTT.

\begin{tabular}{llll}
\hline Congenita & $<5 \%$ dei casi & $\begin{array}{l}\text { Attività ADAMTS13 }<5 \% \text { Mutazione } \\
\text { di ADAMTS13 }\end{array}$ & $\begin{array}{l}\text { Età di presentazione variabile (nascita, } \\
\text { infanzia, adulti, donne gravide) }\end{array}$ \\
\hline $\begin{array}{l}\text { Acquisita } \\
\text { Idiopatica }\end{array}$ & $80 \%$ circa dei casi & $\begin{array}{l}\text { Attività ADAMTS13 }<5 \% \text { all'esordio } \\
\text { (più alta se recidive) } \\
\text { Anticorpi IgG o inibizione }\end{array}$ & $\begin{array}{l}\text { Assenza di cause evidenti ma possibile } \\
\text { trigger (per esempio infezioni) }\end{array}$ \\
\hline Secondaria & $10-15 \%$ dei casi & $\begin{array}{l}\text { Attività ADAMTS13 e titolo anticorpale } \\
\text { variabile }\end{array}$ & Patologie predisponenti e/o concomitanti \\
\hline
\end{tabular}

* Si veda tabella 2 
Tabella 2 Cause predisponenti e/o concomitanti di PTT secondaria.

- Anemia emolitica autoimmune

- Coagulazione intravascolare disseminata (CID)

- Infezione/sepsi

- Neoplasia; stomaco, mammella, polmone

- Farmaci: inibitori della calcineurina, ciclosporina A, estroprogestinici, valaciclovir, chinidina, chemioterapici (mitomicina C, bleomicina, cisplatino, gemcitabina), ticlopidina

- Gravidanza e post-partum

- Malattie autoimmuni: sindrome da anticorpi antifosfolipidi, lupus eritematoso sistemico, sclerodermia

- Ipertensione maligna

- AIDS

- Device o "corpi estranei"

- Diarrea causata da Escherichia coli enteroemorragico

- Chirurgia maggiore

caso di sospensione del trattamento antiretrovirale [19]. Stranamente, i soggetti con attività di ADAMTS13 > $\%$ presentano anche un aumento di mortalità rispetto a quelli con attività $<5 \%$ [20].

Il trattamento prevede, come prima scelta, la plasmaferesi in associazione alla terapia antiretrovirale con l'obiettivo di ottenere la normalizzazione dei livelli di ADAMTS13 e la riduzione della carica virale [19]. Nei casi refrattari alla terapia standard, come nei pazienti non affetti da HIV, può essere considerato un successivo trattamento con rituximab. La somministrazione di steroidi non sembra associarsi a un aumento significativo di infezioni opportunistiche.

Sono stati segnalati, inoltre, casi di PTT secondaria all'impianto di device (apparecchio ortodontico fisso, dispositivo contraccettivo intrauterino) per cui è stata necessaria, in associazione alla terapia convenzionale, la rimozione del device stesso [21].

Riguardo alla gravidanza, i periodi a maggior rischio per lo sviluppo di PTT sono rappresentati dal terzo trimestre gestazionale e dal post-partum [22].

La gravidanza costituisce il $10-25 \%$ circa delle cause scatenanti una PTT nelle donne. Il 45\% circa delle donne che sviluppano una PTT è in età fertile. Appare inoltre aumentato il rischio di sviluppare una recidiva di PTT o altre microangiopatie trombotiche nelle gravidanze successive. Nelle donne sane è presente una fisiologica e progressiva riduzione dell'attività di ADAMTS13 dal terzo trimestre, con un ritorno a valori normali nel post-partum. È possibile distinguere due forme di PTT durante la gravidanza: la PTT congenita a insorgenza tardiva e quella acquisita mediata da anticorpi. La forma congenita è caratterizzata da un rischio più alto di recidiva nelle gravidanze successive rispetto alla forma acquisita. La diagnosi è confermata dall'attività di ADAMTS13 $<5 \%$, dall'assenza di anticorpi e dalla presenza di mutazioni all'analisi genetica [23].

Vi è un alto tasso di mortalità fetale $(50 \%)$, causata da ischemia placentare secondaria a trombosi delle arteriole decidue, e morbilità (46\% di neonati prematuri) [24].

Nonostante la plasmaferesi induca la rimozione degli ormoni che permettono il mantenimento della gravidanza, rimane il trattamento di scelta.
Non viene più annoverato tra le cause di PTT il trapianto di midollo osseo: si preferisce parlare di "microangiopatia associata al trapianto" (MAT). È dovuta a danno endoteliale secondario alla chemioterapia, infezioni, immunosoppressione e graft versus host disease. Clinicamente presenta caratteristiche distintive: non risponde alla plasmaferesi e sono assenti formazioni microtrombotiche sistemiche [23].

\section{Diagnosi}

Per la diagnosi di PTT è sufficiente il riscontro di anemia emolitica microangiopatica e piastrinopenia in assenza di altre cause evidenti $[25,26]$. La presenza degli altri tre elementi della classica pentade (insufficienza renale acuta, alterazioni neurologiche e febbre) non è più necessaria per la diagnosi, trattandosi di segni di danno d'organo avanzato.

\section{Sintomi}

- Manifestazioni emorragiche: petecchie, ecchimosi, porpora, epistassi (tabella 3).

- Manifestazioni trombotiche: neurologiche, renali, cardiovascolari.

Le manifestazioni neurologiche si distinguono in frequenti (cefalea, alterazione dello stato mentale) e rare: attacco ischemico transitorio, ictus, coma, convulsioni, deficit motori, disturbi del visus, crisi di grande male.

Le manifestazioni renali comprendono oliguria, anuria, insufficienza renale acuta (raramente con necessità di trattamento dialitico).

Riguardo alle manifestazioni cardiovascolari, la formazione di microtrombi associati a emorragie può coinvolgere tutto il tessuto cardiaco, le coronarie e il sistema di conduzione elettrico inducendo l'insorgenza di aritmie, morte cardiaca improvvisa, infarto miocardico acuto, insufficienza cardiaca sino allo shock $[27,28]$.

- Sintomi vari: febbre, diarrea, vomito, dolori addominali.

\section{Dati di laboratorio}

La diagnosi clinica deve essere confermata dai seguenti dati di laboratorio.

- Trombocitopenia: conta piastrinica $<5010^{9} / \mathrm{L}$.

- Anemia emolitica microangiopatica: è definita dalla presenza di emolisi non indotta da anticorpi associata alla presenza di schistociti all'esame dello striscio del sangue periferico; ematocrito < 20\%; emoglobina $<10 \mathrm{~g} / \mathrm{dL}$; aumento della bilirubina indiretta. La frammentazione dei globuli rossi può indurre una progressiva riduzione del valore del volume corpuscolare (MCV) tale che i lettori automatici possono erroneamente considerare gli schistociti come piastrine, sottostimando in questo modo la piastrinopenia. Un valore di schistociti $\geq 1 \%$ è attualmente considerato suggestivo per la diagnosi di PTT $[29,30]$. Più raramente, in una fase molto precoce di malattia, come può accadere nei pazienti sottoposti a stretto monitoraggio clinico-laboratoristico dopo un precedente episodio di PTT, la diagnosi è possibile in assenza di frammentazione evidente dei globuli rossi allo striscio periferico [31]. 
Tabella 3 Sintomi di presentazione ed esami di laboratorio alla diagnosi di PTT.

\begin{tabular}{|c|c|}
\hline \multicolumn{2}{|l|}{ Sintomi } \\
\hline \multicolumn{2}{|l|}{ - Manifestazioni emorragiche: petecchie, ecchimosi, porpora, epistassi } \\
\hline \multicolumn{2}{|c|}{$\begin{array}{l}\text { - Manifestazioni trombotiche } \\
\text { - Sistema nervoso } \\
\text { - Frequenti: cefalea, alterazione dello stato mentale } \\
\text { - Rare: attacco ischemico transitorio, ictus, coma, convulsioni, deficit motori, disturbi del visus, crisi di grande male } \\
\text { - Rene: oliguria, anuria, insufficienza renale acuta } \\
\text { - Cuore: aritmie, morte cardiaca improvvisa, infarto miocardico acuto, insufficienza cardiaca, shock }\end{array}$} \\
\hline \multicolumn{2}{|c|}{ - Vari: febbre, diarrea, vomito, dolori addominali } \\
\hline \multicolumn{2}{|c|}{ Esami di laboratorio } \\
\hline Emocromo ed esame dello striscio periferico & Anemia, piastrinopenia, schistociti \\
\hline Conta dei reticolociti & Aumentata \\
\hline Aptoglobina & Ridotta \\
\hline Lattato-deidrogenasi & Aumentata \\
\hline Coagulazione: tempo di protrombina, tempo di protrombina parziale & Normali \\
\hline Fibrinogeno, D-dimero & Normali o aumentati \\
\hline Troponina & Normale o aumentata \\
\hline Funzionalità renale: elettroliti & Normali o alterati \\
\hline Esame delle urine e urinocoltura & Normali o alterate \\
\hline Funzionalità epatica/bilirubina totale e frazionata & Normale o alterata/aumentata \\
\hline Test di Coombs diretto e indiretto & Negativi \\
\hline \multicolumn{2}{|l|}{ Sierologia per epatite A, B, C e HIV } \\
\hline \multicolumn{2}{|l|}{ Test di gravidanza } \\
\hline \multicolumn{2}{|l|}{ Gruppo sanguigno } \\
\hline \multicolumn{2}{|c|}{ Screening immunologico: anticorpi antinucleo, fattore reumatoide, anticoagulante lupico, anticorpi anticardiolipina } \\
\hline Dosaggio dell'attività di ADAMTS13 e titolo anticorpale & \\
\hline
\end{tabular}

Sono inoltre presenti reticolocitosi, livelli ridotti o indosabili di aptoglobina, elevati livelli sierici di lattato-deidrogenasi (LDH) generati dall'emolisi stessa e dal danno ischemico tissutale.

I test di Coombs, diretto e indiretto, risultano negativi. Il tempo di protrombina e il tempo di tromboplastina parziale sono di solito nella norma, mentre il fibrinogeno, proteina di fase acuta, risulta aumentato.

L'esame delle urine evidenzia spesso una lieve proteinuria (1-2 g/die) e poche cellule o cilindri. Talora il reperto si pone in diagnosi differenziale con una glomerulonefrite o una vasculite, soprattutto se nel sedimento urinario sono presenti globuli rossi o cilindri ematici associati a ipocomplementemia (riscontrata in circa la metà dei pazienti), più raramente associate però a piastrinopenia marcata come nel caso della PTT.

In circa un quarto dei pazienti, l'evento acuto progredisce verso l'insufficienza renale cronica. In un recente studio, il riscontro di alti livelli di troponina I all'esordio, indice di coinvolgimento cardiaco, correla con un aumento della mortalità e morbilità in fase acuta [32].

Altri esami di laboratorio raccomandati nel sospetto di PTT all'esordio sono lo studio della funzionalità epatica, test sierologici per le epatiti e l'HIV, lo screening immunologico (anticorpi antinucleo, fattore reumatoide, anticoagulante lupico, anticorpi anticardiolipina) (tabella 3).

\section{ADAMTS13}

Tra i pazienti con PTT idiopatica, la percentuale con un severo deficit di ADAMTS13 (attività $<5 \%$ ) varia tra il $33 \%$ e il $100 \%[9,10,33-35]$.

Al contrario, nei pazienti con PTT secondaria il deficit di ADAMTS13 è spesso assente o risulta lieve. La riduzione dei livelli di ADAMTS13 è stata documentata anche in diverse condizioni patologiche non associate a PTT [36] tra cui coagulazione intravascolare disseminata (CID), porpora trombocitopenica idiopatica, infezioni/sepsi [37-39], neoplasie $[40,41]$, trattamento con chemioterapici, piastrinopenia indotta da eparina (HIT), trapianto di midollo osseo, sindrome da anticorpi antifosfolipidi, lupus eritematoso sistemico [42], cirrosi [43], uremia, dopo interventi chirurgici, nei soggetti sani e nei neonati [36].

Diversi studi hanno evidenziato una relazione inversa tra i livelli sierici della proteasi e l'antigene del VWF e la sua attività, suggerendo che i livelli di ADAMTS13 si riducono come risposta alla liberazione di multimeri ULVWF nel circolo. Ciò potrebbe spiegare la diminuzione dei valori dell'attività di 
ADAMTS13 durante gli eventi stressanti, con induzione di una risposta infiammatoria, che è stata associata all'insorgenza di PTT acuta.

I dosaggi di ADAMTS13 possono essere alterati dalle trasfusioni eventualmente somministrate per trattare l'evento acuto, considerando che l'emivita plasmatica di ADAMTS13 è di 2-3 giorni.

Il deficit congenito di ADAMTS13 non sempre si presenta durante l'infanzia e questo rende difficile la diagnosi differenziale con i pazienti affetti da PTT acquisita che non rispondono all'infusione di plasma. In alcuni casi il riscontro di anticorpi anti-ADAMTS13 può aiutare nell'identificazione.

Nella maggior parte dei pazienti affetti da PTT acquisita, che non presentano un severo deficit di ADAMTS13, la causa della malattia non è nota.

Inoltre, sono pochi i laboratori in grado di fornire una risposta in tempi rapidi sul deficit di ADAMTS13, pertanto il clinico deve sospettare la diagnosi e iniziare la terapia in assenza di tale informazione.

\section{ADAMTS13 e prognosi}

In generale, i pazienti con PTT idiopatica rispondono alla terapia con plasmaferesi, quelli con PTT secondaria no.

Pur considerando che il deficit di ADAMTS13 è molto raro nei pazienti affetti da PTT secondaria, tale deficit correla con una migliore risposta alla plasmaferesi [33,34].

Nella PTT idiopatica l'utilità di distinguere i pazienti con o senza deficit di ADAMTS13 è ancora discussa, ma sembra certa la correlazione tra il livello del deficit e la prognosi [36].

Diversi studi hanno posto attenzione alla correlazione tra i dosaggi di ADAMTS13 alla diagnosi e la risposta alla terapia con plasmaferesi, la frequenza di recidiva e la sopravvivenza. I pazienti con o senza deficit di ADAMTS13 presentano un tasso di sopravvivenza simile (rispettivamente $80 \%$ e $90 \%$ ) nel breve periodo. I pazienti con un severo deficit di ADAMTS13 o con anticorpi anti-ADAMTS13 positivi durante la fase di remissione dimostrano un tasso di recidiva significativamente più elevato (in media il $30 \%$ circa), mentre i pazienti senza un deficit severo recidivano nel $9 \%$ circa dei casi $[33,34,44]$.

Visto il breve follow-up della maggior parte di questi studi, il rischio è di sottostimare, nel deficit severo di ADAMTS13, la percentuale di recidive, che tende ad aumentare nel corso degli anni. In uno studio condotto presso l'Università di Washington, in un iniziale follow-up compreso tra 8 e 33 mesi, su 16 pazienti affetti da PTT idiopatica con severo deficit di ADAMTS13, il tasso di recidiva era del 38\% (6 pazienti) [34]. A distanza di tre anni, 11 (69\%) dei 16 pazienti hanno presentato almeno un episodio di recidiva di malattia, con un periodo di benessere compreso tra 8 mesi e 5 anni, e 5 (31\%) di loro sono deceduti.

Il dosaggio degli anticorpi anti-ADAMTS13 fornisce ulteriori informazioni prognostiche.

In diversi studi la presenza di anticorpi alla diagnosi correla con un rischio più alto di recidiva di malattia $[33,35]$. Un alto titolo anticorpale correla inoltre con scarsa risposta alla plasmaferesi, malattia refrattaria e morte precoce [45].

Tuttavia tali risultati si basano su studi con pochi pazienti e alcuni di essi non hanno osservato una relazione tra il titolo degli anticorpi anti-ADAMTS13 e l'outcome a breve termine [46]. Pur considerando l'estrema variabilità dei dosaggi dell'attività di ADAMTS13 e del titolo anticorpale nel corso della malattia, si è osservato che un singolo test al momento della diagnosi è in grado di prevedere il rischio di una successiva recidiva. I livelli di ADAMTS13 durante la fase di remissione correlano invece con l'outcome clinico. In un recente studio su 109 pazienti che presentavano una completa risposta alla plasmaferesi, il riscontro di recidive si aveva in circa il $60 \%$ nei pazienti con deficit severo e nel $19 \%$ in quelli senza deficit [47].

In conclusione, sembra evidente che i pazienti affetti da PTT idiopatica causata da deficit di ADAMTS13 tendono a presentare una recidiva che risulta associata alla persistenza o ricorrenza del deficit severo di ADAMTS13. Un monitoraggio laboratoristico al termine della terapia con plasmaferesi potrebbe essere utile per identificare i pazienti con deficit di ADAMTS13 ad alto rischio di recidiva a breve termine.

\section{Diagnosi differenziale}

La PTT entra in diagnosi differenziale con altre sindromi caratterizzate dalla presenza di trombocitopenia associata ad anemia emolitica microangiopatica quale la sindrome emolitico-uremica (HUS), la coagulazione intravascolare disseminata (CID), la sindrome "catastrofica" da antifosfolipidi (APS), la sindrome HELLP (Hemolysis, Elevated Liver function and Low Platelets), la trombocitopenia indotta da eparina (HIT).

I principali dati clinici e di laboratorio utili per differenziare la PTT dalle altre microangiopatie trombotiche sono riassunti in tabella 4.

\section{Terapia}

\section{Plasmaferesi}

Il trattamento attuale della PTT prevede l'utilizzo della plasmaferesi $(40-60 \mathrm{~mL} / \mathrm{kg} / \mathrm{die})$ [48]. Nel caso in cui non fosse possibile, i pazienti dovrebbero essere trattati con l'infusione di plasma sino a $30 \mathrm{~mL} / \mathrm{kg} /$ die valutando nel singolo paziente la tolleranza al carico di fluidi [25].

Questi approcci terapeutici riducono la mortalità dall'80$90 \%$ al $10 \%$ [4].

Il meccanismo alla base dell'efficacia della terapia con plasma exchange è dovuto alla rimozione dal circolo degli anticorpi anti-ADAMTS13 nelle forme immunomediate e dalla correzione della carenza della proteasi nelle forme congenite [49].

È stato dimostrato che un ritardo nell'inizio del trattamento (dopo le 24 ore) può comprometterne l'efficacia. Sono dunque importanti la rapidità nel porre la diagnosi di PTT e l'inizio del trattamento con plasmaferesi a diagnosi clinica formulata o sospettata. Nel caso di una diagnosi alternativa la plasmaferesi può essere sospesa.

Nel caso di PTT secondaria a diarrea nei bambini o a chemioterapia non ci sono evidenze di beneficio con il trattamento aferetico, che può in ogni caso essere intrapreso se è presente un severo interessamento neurologico. Non vi sono indicazioni circa il numero esatto di sedute di plasma 
Tabella 4 Diagnosi differenziale tra PTT e altre microangiopatie trombotiche.

\begin{tabular}{|c|c|c|}
\hline Patologia & Sintomi e segni comuni & Sintomi e segni differenziali \\
\hline $\begin{array}{l}\text { Sindrome emolitico-uremica } \\
\text { (HUS) }\end{array}$ & $\begin{array}{l}\text { Anemia emolitica } \\
\text { Trombocitopenia } \\
\text { Anemia con schistociti }\end{array}$ & $\begin{array}{l}\text { - Infezione gastrointestinale: Escherichia coli 0157:H7, } \\
\text { Shigella dysenteriae } \\
\text { - Diarrea con melena } \\
\text { - Incremento dei livelli sierici di azotemia e creatininemoa } \\
\text { - Segni di coagulazione intravascolare disseminata (CID) } \\
\text { compensata }\end{array}$ \\
\hline $\begin{array}{l}\text { Coagulazione intravascolare } \\
\text { disseminata (CID) }\end{array}$ & $\begin{array}{l}\text { Anemia emolitica } \\
\text { Trombocitopenia }\end{array}$ & $\begin{array}{l}\text { - Marcato incremento dei prodotti di degradazione del fibrinogeno } \\
\text { (FDP e D-dimero) } \\
\text { - Ipofibrinogenemia } \\
\text { - Prolungamento dei tempi di protrombina e tromboplastina } \\
\text { parzialmente attivata }\end{array}$ \\
\hline $\begin{array}{l}\text { Sindrome HELLP (Hemolysis, } \\
\text { Elevated Liver function and } \\
\text { Low Platelets) }\end{array}$ & $\begin{array}{l}\text { Anemia emolitica } \\
\text { Trombocitopenia }\end{array}$ & - Incremento degli enzimi epatici \\
\hline Preeclampsia, eclampsia & $\begin{array}{l}\text { Trombocitopenia } \\
\text { Proteinuria }\end{array}$ & $\begin{array}{l}\text { - Ipertensione di nuova diagnosi (seconda metà del periodo } \\
\text { gestazionale) } \\
\text { - Edema periferico } \\
\text { - Incremento dei valori di PAI-1 } \\
\text { - Incremento dei prodotti di degradazione del fibrinogeno } \\
\text { (FDP e D-dimero) }\end{array}$ \\
\hline $\begin{array}{l}\text { Sindrome catastrofica da } \\
\text { anticorpi antifosfolipidi (APS) }\end{array}$ & Trombocitopenia & $\begin{array}{l}\text { - Presenza di anticorpi antinucleo e anticardiolipina } \\
\text { - Positività della ricerca del anticoagulante lupico }\end{array}$ \\
\hline Sindrome di Evans & $\begin{array}{l}\text { Anemia emolitica } \\
\text { Trombocitopenia }\end{array}$ & $\begin{array}{l}\text { - Presenza concomitante di anticorpi antipiastrine e antieritrociti } \\
\text { - Test di Coombs positivo } \\
\text { - Assenza di schistociti e di sindrome ischemica degli organi } \\
\text { periferici }\end{array}$ \\
\hline $\begin{array}{l}\text { Trombocitopenia indotta da } \\
\text { eparina (HIT) }\end{array}$ & Trombocitopenia & - Assenza di emolisi e schistociti \\
\hline
\end{tabular}

exchange necessarie a ottenere la remissione della fase acuta. L'orientamento è di proseguire sino al raggiungimento della remissione dei sintomi in presenza di conta piastrinica stabilmente $>15010^{\circ} / \mathrm{L}$, normalizzazione dei livelli sierici di LDH e correzione dell'anemia. Meno definita è l'indicazione a proseguire la plasmaferesi dopo la normalizzazione degli esami di laboratorio [50]. Alcuni autori hanno suggerito di proseguire per ulteriori 3 giorni dopo il raggiungimento della remissione [51]. Diverse sono le complicanze legate alla procedura e alla necessità di un accesso venoso centrale: pneumotorace, emotorace, sepsi, shock anafilattico, tamponamento cardiaco [4]. Taluni autori riportano l'utilità della profilassi per gli episodi ricorrenti nella PTT congenita, basata sulla somministrazione di plasma $(30 \mathrm{~mL} / \mathrm{kg})$ a intervalli regolari (ogni 5-7 giorni) [51].

\section{Corticosteroidi}

Per la forma acquisita di PTT, causata dalla presenza di autoanticorpi anti-ADAMTS13, è stata proposta l'associazione di una terapia immunosoppressiva alla terapia con plasma exchange. Spesso, però, l'informazione sul titolo anticorpale non è disponibile in tempi sufficientemente rapidi. Appare quindi razionale l'utilizzo di glucocorticoidi nei pazienti affetti da PTT in cui è ipotizzabile un'eziologia autoimmune. Pur non essendo disponibili studi randomizzati sull'efficacia di questa strategia terapeutica, prednisone $(1,0 \mathrm{mg} / \mathrm{kg} / \mathrm{die})$ o metilprednisolone $(125 \mathrm{mg} 2$ volte $/$ die $)$ possono essere presi in considerazione in caso di una scarsa risposta al trattamento iniziale con plasmaferesi $[52,30]$ e nei pazienti in cui la conta piastrinica non risalga dopo diversi giorni di plasmaferesi o torni a scendere se le sedute vengono ridotte o sospese [26]. La sola terapia con glucocorticoidi non è raccomandata. L'efficacia del trattamento di prevenzione di recidiva nelle forme croniche ricorrenti di PTT mediante somministrazione di basse dosi di corticosteroidi non è stata dimostrata, tuttavia questo approccio terapeutico è frequentemente adottato.

\section{Rituximab}

Negli ultimi anni vi è stato un incremento nell'utilizzo degli anticorpi monoclonali anti-CD20 (rituximab) nella terapia della PTT, in particolare nei casi refrattari al trattamento con plasma exchange che presentano sintomi neurologici o caratterizzati da frequenti episodi ricorrenti.

La malattia refrattaria è stata definita arbitrariamente dalla presenza di piastrinopenia persistente (conta piastrinica $\left.<15010^{9} / \mathrm{L}\right)$ o LDH aumentata dopo 7 sedute di plasmaferesi associate o no a corticosteroidi. 
Lo scopo del trattamento con rituximab è sopprimere la produzione degli anticorpi anti-ADAMTS13 mediante deplezione dei linfociti $B$. La dose raccomandata di rituximab è di $375 \mathrm{mg} / \mathrm{m}^{2}$ ogni 7 giorni, ripetuta per 3 o 4 cicli [53,54]. Diversi case report e lavori su piccole casistiche in pazienti affetti da PTT idiopatica, refrattaria ai trattamenti con plasma exchange e corticosteroidi, oppure recidivante, suggeriscono che il rituximab sia in grado di indurre una risposta completa, clinica e di laboratorio, nel 95\% circa dei soggetti affetti in 1-3 settimane dall'inizio del trattamento. La risposta al rituximab è correlata alla scomparsa degli anticorpi anti-ADAMTS13 con normalizzazione dei livelli plasmatici dell'enzima.
Reazioni acute di lieve entità, secondarie all'infusione di rituximab, sono controllate con la somministrazione preventiva di corticosteroidi, antistaminici e analgesici. Reazioni avverse più gravi sono meno frequenti, anche se sono stati riportati un caso di shock cardiogeno [55] e un altro di infezione gastrointestinale sintomatica da Strongyloides [53].

La recidiva si è presentata in circa il $10 \%$ dei pazienti a distanza di 9 mesi-4 anni e tutti, eccetto uno, hanno risposto a un secondo trattamento con rituximab cui è seguito un ulteriore, lungo periodo di remissione completa $[53,54,56]$.

$\grave{E}$ attualmente in corso uno studio multicentrico di fase II per meglio definire il ruolo del rituximab nella strategia terapeutica della PTT [57].

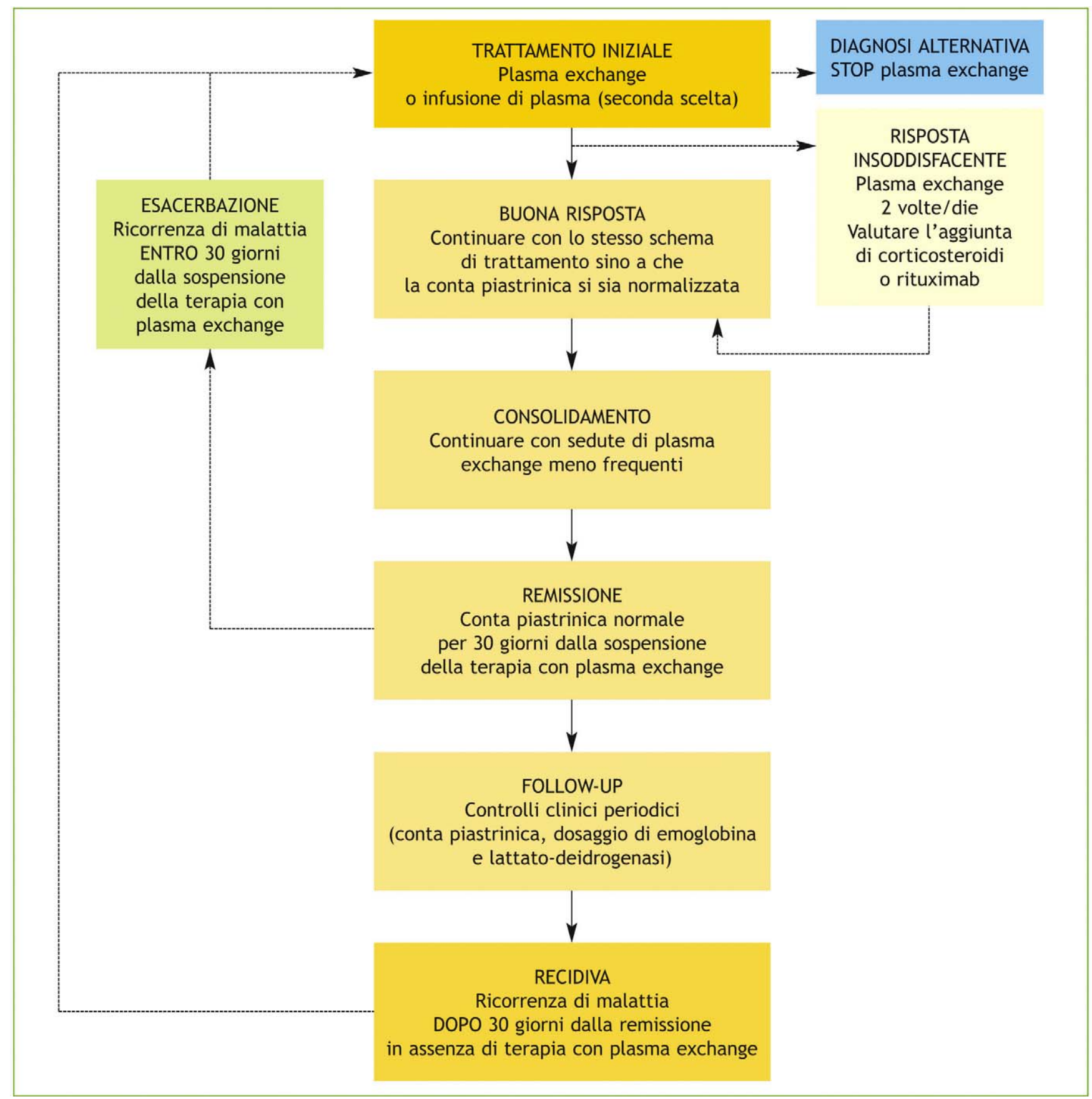

Figura 2 Algoritmo terapeutico della PTT. 


\section{Altre terapie}

La ciclosporina non è solitamente utilizzata nel trattamento della PTT a causa della possibile tossicità sull'endotelio. Tuttavia, alcuni studi hanno evidenziato una possibile efficacia della ciclosporina (300 mg/die per os) nei pazienti che presentano una forma recidivante o refrattaria alla plasmaferesi (associata ai corticosteroidi) o non responsiva al trattamento con rituximab [58].

Con le stesse indicazioni sono riportati casi di impiego della vincristina [59].

La somministrazione endovenosa di immunoglobuline (IVIG) potrebbe risultare efficace, anche se non alternativa alla plasmaferesi. Il meccanismo con cui le IVIG agirebbero non è chiaro. L'ipotesi, pur non essendo disponibili dati, è che agiscano legandosi e inattivando alcune proteine coinvolte nell'aggregazione piastrinica. La dose utilizzata nel trattamento con immunoglobuline per via endovenosa è pari a $400 \mathrm{mg} / \mathrm{kg}$ per 5 giorni o in alternativa $1 \mathrm{~g} / \mathrm{kg}$ per 2 giorni.

La splenectomia, come in altre patologie su base autoimmune, può essere effettivamente presa in considerazione nelle forme di PTT cronica ricorrente refrattaria agli altri approcci terapeutici. La sua efficacia, che tuttavia non è costante, si basa sulla rimozione di un'importante sede di produzione degli autoanticorpi anti-ADAMTS13.

Gli inibitori dell'aggregazione piastrinica quali la ticlopidina, il clopidogrel, l'acido acetilsalicilico e il dipiridamolo non hanno indicazioni nel trattamento della PTT [4] non essendo in grado di inibire l'aggregazione piastrinica indotta dai multimeri ULVWF [6]. Utile è la terapia di supporto con emotrasfusioni, supplementi di acido folico ed eventuale profilassi antitrombotica con eparina a basso peso molecolare (se conta piastrinica $>5010^{9} / \mathrm{L}$ ).

\section{Follow-up}

Dopo la sospensione del trattamento aferetico, i pazienti devono essere sottoposti a frequenti controlli che prevedono un emocromo completo e il dosaggio della LDH. Se i valori si mantengono nella norma, l'intervallo tra i successivi controlli può essere prolungato.

La recidiva, definita come ricomparsa di PTT dopo almeno 30 giorni di remissione completa in assenza di trattamento, è comune nei pazienti con un severo deficit di ADAMTS13 che non rispondono alla terapia iniziale. La comparsa di recidiva avviene frequentemente entro il primo anno nei pazienti con un'attività di ADAMTS13 $<10 \%$ e presenza di anticorpi antiADAMTS13 [47].

Nei pazienti con un deficit severo di ADAMTS13 il rischio di recidiva è del $40 \%$ circa [4]. In tutti sono raccomandati, in ogni caso, un monitoraggio continuo e un tempestivo controllo della conta piastrinica nel sospetto di una ripresa di malattia.

\section{Terapia di mantenimento}

Non è nota l'efficacia di una terapia di mantenimento per la prevenzione delle recidive nelle forme di PTT idiopatica.

Nelle forme congenite è invece risultato efficace un trattamento di profilassi delle recidive con piccoli volumi di plasma fresco congelato (10 mL/kg ogni 2-4 settimane) [60].
Un possibile algoritmo per il trattamento della PTT [30], successivamente modificato, è proposto in fig. 2 .

\section{Conflitto di interesse}

Gli autori dichiarano di essere esenti da conflitto di interessi.

\section{Bibliografia}

[1] Miller DP, Kaye JA, Shea K, et al. Incidence of thrombotic thrombocytopenic purpura/hemolytic uremic syndrome. Epidemiology 2004;15(2):208-15.

[2] Terrell DR, Williams LA, Vesely SK, Lämmle B, Hovinga JA, George JN. The incidence of thrombotic thrombocytopenic purpura-hemolytic uremic syndrome: all patients, idiopathic patients, and patients with severe ADAMTS-13 deficiency. J Thromb Haemost 2005;3(7):1432-6.

[3] Schech SD, Brinker A, Shatin D, Burgess M. New-onset and idiopathic thrombotic thrombocytopenic purpura: incidence, diagnostic validity, and potential risk factors. Am J Hematol 2006;81(9):657-63.

[4] George JN. The thrombotic thrombocytopenic purpura and hemolytic uremic syndromes: evaluation, management, and long-term outcomes experience of the Oklahoma TTP-HUS Registry, 1989-2007. Kidney Int Suppl 2009;112:S52-4.

[5] Moschowitz E. Hyaline thrombosis of the terminal arterioles and capillaries: a hitherto undescribed disease. Proc N Y Pathol Soc 1924;24:21-4.

[6] Moake JL, Rudy CK, Troll JH, et al. Unusually large plasma factor VIII:von Willebrand factor multimers in chronic relapsing thrombotic thrombocytopenic purpura. N Engl J Med 1982;307(23): 1432-5.

[7] Furlan M, Robles R, Lämmle B. Partial purification and characterization of a protease from human plasma cleaving von Willebrand factor to fragments produced by in vivo proteolysis. Blood 1996;87(10):4223-34.

[8] Tsai HM. Physiologic cleavage of von Willebrand factor by a plasma protease is dependent on its conformation and requires calcium ion. Blood 1996;87(10):4235-44.

[9] Furlan M, Robles R, Galbusera M, et al. von Willebrand factorcleaving protease in thrombotic thrombocytopenic purpura and the hemolytic-uremic syndrome. N Engl J Med 1998;339(22): 1578-84.

[10] Tsai HM, Lian EC. Antibodies to von Willebrand factor-cleaving protease in acute thrombotic thrombocytopenic purpura. N Engl J Med 1998;339(22):1585-94.

[11] Fujikawa K, Suzuki H, McMullen B, Chung D. Purification of human von Willebrand factor-cleaving protease and its identification as a new member of the metalloproteinase family. Blood 2001;98(6):1662-6.

[12] Gerritsen HE, Robles R, Lämmle B, Furlan M. Partial amino acid sequence of purified von Willebrand factor-cleaving protease. Blood 2001;98(6):1654-61.

[13] Zheng X, Chung D, Takayama TK, Majerus EM, Sadler JE, Fujikawa K. Structure of von Willebrand factor-cleaving protease (ADAMTS13), a metalloprotease involved in thrombotic thrombocytopenic purpura. J Biol Chem 2001;276(44): 41059-63.

[14] Levy GG, Nichols WC, Lian EC, et al. Mutations in a member of the ADAMTS gene family cause thrombotic thrombocytopenic purpura. Nature 2001;413(6855):488-94.

[15] Shelat SG, Smith P, Ai J, Zheng XL. Inhibitory autoantibodies against ADAMTS-13 in patients with thrombotic thrombocytopenic purpura bind ADAMTS-13 protease and may accelerate its clearance in vivo. J Thromb Haemost 2006;4(8):1707-17. 
[16] Kojouri K, Vesely SK, George JN. Quinine-associated thrombotic thrombocytopenic purpura-hemolytic uremic syndrome: frequency, clinical features, and long-term outcomes. Ann Intern Med 2001;135(12):1047-51.

[17] Tsai HM, Rice L, Sarode R, Chow TW, Moake JL. Antibody inhibitors to von Willebrand factor metalloproteinase and increased binding of von Willebrand factor to platelets in ticlopidine-associated thrombotic thrombocytopenic purpura. Ann Intern Med 2000;132(10):794-9.

[18] Bennett CL, Kim B, Zakarija A, et al., SERF-TTP Research Group. Two mechanistic pathways for thienopyridine-associated thrombotic thrombocytopenic purpura: a report from the SERF-TTP Research Group and the RADAR Project. J Am Coll Cardiol 2007;50(12):1138-43.

[19] Miller RF, Scully M, Cohen H, et al. Thrombotic thrombocytopaenic purpura in HIV-infected patients. Int J STD AIDS 2005;16(8):538-42.

[20] Malak S, Wolf M, Millot GA, et al. Réseau d'Etude des Microangiopathies Thrombotiques (TMA-Rare Diseases Reference Center). Human immunodeficiency virus-associated thrombotic microangiopathies: clinical characteristics and outcome according to ADAMTS13 activity. Scand J Immunol 2008;68(3):33744.

[21] Anselmi E, Arcari A, Bernuzzi P, et al. Thrombotic thrombocytopenic purpura: report of seven cases. Ann Ital Med Int 2005;20(2):108-12.

[22] George JN. The association of pregnancy with thrombotic thrombocytopenic purpura-hemolytic uremic syndrome. Curr Opin Hematol 2003;10(5):339-44.

[23] Scully MA, Machin SJ. Berend Houwen Memorial Lecture: ISLH Las Vegas May 2009: the pathogenesis and management of thrombotic microangiopathies. Int J Lab Hematol 2009;31(3): 268-76.

[24] Fujimura Y, Matsumoto M, Kokame K, et al. Pregnancy-induced thrombocytopenia and TTP, and the risk of fetal death, in Upshaw-Schulman syndrome: a series of 15 pregnancies in 9 genotyped patients. Br J Haematol 2009;144(5):742-54.

[25] Rock GA, Shumak KH, Buskard NA, et al. Comparison of plasma exchange with plasma infusion in the treatment of thrombotic thrombocytopenic purpura. Canadian Apheresis Study Group. N Engl J Med 1991;325(6):393-7.

[26] George JN. Clinical practice. Thrombotic thrombocytopenic purpura. N Engl J Med 2006;354(18):1927-35.

[27] Patschan D, Witzke O, Dührsen U, Erbel R, Philipp T, HergetRosenthal $S$. Acute myocardial infarction in thrombotic microangiopathies - clinical characteristics, risk factors and outcome. Nephrol Dial Transplant 2006;21(6):1549-54.

[28] Gami AS, Hayman SR, Grande JP, Garovic VD. Incidence and prognosis of acute heart failure in the thrombotic microangiopathies. Am J Med 2005;118(5):544-7.

[29] Burns ER, Lou Y, Pathak A. Morphologic diagnosis of thrombotic thrombocytopenic purpura. Am J Hematol 2004;75(1):18-21.

[30] George JN. How I treat patients with thrombotic thrombocytopenic purpura-hemolytic uremic syndrome. Blood 2000;96(4): 1223-9.

[31] Fava S, Galizia AC. Thrombotic thrombocytopenic purpura-like syndrome in the absence of schistocytes. $\mathrm{Br} \mathrm{J}$ Haematol 1995;89(3):643-4.

[32] Hughes C, McEwan JR, Longair I, et al. Cardiac involvement in acute thrombotic thrombocytopenic purpura: association with troponin T and IgG antibodies to ADAMTS 13. J Thromb Haemost 2009;7(4):529-36.

[33] Vesely SK, George JN, Lämmle B, et al. ADAMTS13 activity in thrombotic thrombocytopenic purpura-hemolytic uremic syndrome: relation to presenting features and clinical outcomes in a prospective cohort of 142 patients. Blood 2003;102(1):60-8.

[34] Zheng XL, Kaufman RM, Goodnough LT, Sadler JE. Effect of plasma exchange on plasma ADAMTS13 metalloprotease activity, inhibitor level, and clinical outcome in patients with idiopathic and nonidiopathic thrombotic thrombocytopenic purpura. Blood 2004;103(11):4043-9.

[35] Böhm M, Betz C, Miesbach W, et al. The course of ADAMTS-13 activity and inhibitor titre in the treatment of thrombotic thrombocytopenic purpura with plasma exchange and vincristine. Br J Haematol 2005;129(5):644-52.

[36] Sadler JE. Von Willebrand factor, ADAMTS13, and thrombotic thrombocytopenic purpura. Blood 2008;112(1):11-8.

[37] Scully M, Liesner R, Burgess C, Lawrie A, Machin S. ADAMTS 13 in non-thrombotic thrombocytopaenic purpura conditions. $\mathrm{Br} \mathrm{J}$ Haematol 2008;141(2):262-5.

[38] Dwyre DM, Bell AM, Siechen K, Sethi S, Raife TJ. Disseminated histoplasmosis presenting as thrombotic microangiopathy. Transfusion 2006;46(7):1221-5.

[39] Ono T, Mimuro J, Madoiwa S, et al. Severe secondary deficiency of von Willebrand factor-cleaving protease (ADAMTS13) in patients with sepsis-induced disseminated intravascular coagulation: its correlation with development of renal failure. Blood 2006;107(2):528-34.

[40] Francis KK, Kojouri K, George JN. Occult systemic carcinoma masquerading as thrombotic thrombocytopenic purpura-hemolytic uremic syndrome. Community Oncol 2005;2:339-43.

[41] Francis KK, Kalyanam N, Terrell DR, Vesely SK, George JN. Disseminated malignancy misdiagnosed as thrombotic thrombocytopenic purpura: A report of 10 patients and a systematic review of published cases. Oncologist 2007;12(1):11-9.

[42] George JN, Vesely SK, James JA. Overlapping features of thrombotic thrombocytopenic purpura and systemic lupus erythematosus. South Med J 2007;100(5):512-4.

[43] Uemura $M$, Fujimura $Y$, Matsumoto $M$, et al. Comprehensive analysis of ADAMTS13 in patients with liver cirrhosis. Thromb Haemost 2008;99(6):1019-29.

[44] Raife T, Atkinson B, Montgomery R, Vesely S, Friedman K. Severe deficiency of VWF-cleaving protease (ADAMTS13) activity defines a distinct population of thrombotic microangiopathy patients. Transfusion 2004;44(2):146-50.

[45] Coppo P, Wolf M, Veyradier A, et al., Réseau d'Etude des Microangiopathies Thrombotiques de l'Adulte. Prognostic value of inhibitory anti-ADAMTS13 antibodies in adult-acquired thrombotic thrombocytopenic purpura. Br J Haematol 2006;132(1):66-74.

[46] Ferrari S, Scheiflinger F, Rieger $M$, et al., French Clinical and Biological Network on Adult Thrombotic Microangiopathies. Prognostic value of anti-ADAMTS 13 antibody features (Ig isotype, titer, and inhibitory effect) in a cohort of 35 adult French patients undergoing a first episode of thrombotic microangiopathy with undetectable ADAMTS 13 activity. Blood 2007;109(7): 2815-22.

[47] Peyvandi F, Lavoretano S, Palla R, et al. ADAMTS13 and antiADAMTS13 antibodies as markers for recurrence of acquired thrombotic thrombocytopenic purpura during remission. Haematologica 2008;93(2):232-9.

[48] Szczepiorkowski ZM, Bandarenko N, Kim HC, et al., American Society for Apheresis; Apheresis Applications Committee of the American Society for Apheresis. Guidelines on the use of therapeutic apheresis in clinical practice: evidence-based approach from the Apheresis Applications Committee of the American Society for Apheresis. J Clin Apher 2007;22(3):10675.

[49] Moake JL. Thrombotic microangiopathies. N Engl J Med 2002; 347(8):589-600.

[50] Marques MB. Thrombotic thrombocytopenic purpura and heparin-induced thrombocytopenia: two unique causes of life-threatening thrombocytopenia. Clin Lab Med 2009;29(2):321-38.

[51] Coppo P, Bengoufa D, Veyradier A, et al., Réseau d'Etude des Microangiopathies Thrombotiques de l'Adulte. Severe ADAMTS13 deficiency in adult idiopathic thrombotic microangiopathies defines a subset of patients characterized by various 
autoimmune manifestations, lower platelet count, and mild renal involvement. Medicine (Baltimore) 2004;83(4):233-44.

[52] Allford SL, Hunt BJ, Rose P, Machin SJ, Haemostasis and Thrombosis Task Force, British Committee for Standards in Haematology. Guidelines on the diagnosis and management of the thrombotic microangiopathic haemolytic anaemias. $\mathrm{Br} \mathrm{J}$ Haematol 2003;120(4):556-73.

[53] Scully $M$, Cohen $\mathrm{H}$, Cavenagh J, et al. Remission in acute refractory and relapsing thrombotic thrombocytopenic purpura following rituximab is associated with a reduction in IgG antibodies to ADAMTS-13. Br J Haematol 2007;136(3):451-61.

[54] Heidel F, Lipka DB, von Auer C, Huber C, Scharrer I, Hess G. Addition of rituximab to standard therapy improves response rate and progression-free survival in relapsed or refractory thrombotic thrombocytopenic purpura and autoimmune haemolytic anaemia. Thromb Haemost 2007;97(2):228-33.

[55] Millward PM, Bandarenko N, Chang PP, et al. Cardiogenic shock complicates successful treatment of refractory thrombotic thrombocytopenia purpura with rituximab. Transfusion 2005; 45(9):1481-6.
[56] Patino W, Sarode R. Successful repeat therapy with rituximab for relapsed thrombotic thrombocytopenic purpura. J Clin Apher 2007;22(1):17-20.

[57] Foley SR, Webert K, Arnold DM, et al., Members of the Canada Apheresis Group (CAG). A Canadian phase II study evaluating the efficacy of rituximab in the management of patients with relapsed/refractory thrombotic thrombocytopenic purpura. Kidney Int Suppl 2009;112:S55-8.

[58] Cataland SR, Jin M, Lin S, et al. Cyclosporin and plasma exchange in thrombotic thrombocytopenic purpura: long-term follow-up with serial analysis of ADAMTS13 activity. Br J Haematol 2007;139(3):486-93.

[59] Bobbio-Pallavicini E, Porta C, Centurioni R, et al. Vincristine sulfate for the treatment of thrombotic thrombocytopenic purpura refractory to plasma-exchange. The Italian Cooperative Group for TTP. Eur J Haematol 1994;52(4):222-6.

[60] Loirat C, Veyradier A, Girma JP, Ribba AS, Meyer D. Thrombotic thrombocytopenic purpura associated with von Willebrand factor-cleaving protease (ADAMTS13) deficiency in children. Semin Thromb Hemost 2006;32(2):90-7. 